Revue internationale P.M.E.

Économie et gestion de la petite et moyenne entreprise

Revue

internationale

PME

\title{
Qualité des produits et coordination dans la relation de sous-traitance : une analyse économique de la procédure de certification industrielle
}

\section{Bernard Baudry}

Volume 12, numéro 4, 1999

URI : https://id.erudit.org/iderudit/1008665ar

DOI : https://doi.org/10.7202/1008665ar

Aller au sommaire du numéro

Éditeur(s)

Presses de l’Université du Québec

ISSN

0776-5436 (imprimé)

1918-9699 (numérique)

Découvrir la revue

Citer cette note

Baudry, B. (1999). Qualité des produits et coordination dans la relation de sous-traitance : une analyse économique de la procédure de certification industrielle. Revue internationale P.M.E., 12(4), 57-75.

https://doi.org/10.7202/1008665ar
Résumé de l'article

L'objet de cet article consiste à analyser la procédure de certification industrielle dans la relation de sous-traitance. En partant de l'incomplétude contractuelle liée à IIncertitude inhérente à la qualité des produits, l'auteur s'interroge sur les formes de coordination qui permettent néanmoins la production de l'accord entre le donneur d'ordres et le sous-traitant. L'auteur étudie tout d'abord les limites d'une gestion de la qualité des produits par le contrôle effectué par l'acheteur. Puis on montre que l'acquisition de la certification, analysée comme un signal, fonde la réputation de l'entreprise, cette réputation améliorant le fonctionnement du marché par la confiance que placent les acheteurs auprès des vendeurs. Par ailleurs, la qualité ne peut plus être reliée au seul produit, mais elle englobe la totalité de la relation interfirmes, et, de ce fait, la coordination se complexifie. En ce sens, la procédure de certification souffre de nombreuses limites et ne résume pas la totalité des mécanismes de coordination dans la relation de sous-traitance. 
Notes de recherche

\title{
Qualité des produits et coordination dans la relation de sous-traitance: une analyse économique de la procédure de certification industrielle
}

Bernard BAUDRY

Université Lumière Lyon II

MOTS CLÉS

Certification - Sous-traitance - Coordination - Coopération

Réputation - Confiance - Classification JEL : L 15

\begin{abstract}
RÉSUMÉ
L'objet de cet article consiste à analyser la procédure de certification industrielle dans la relation de sous-traitance. En partant de l'incomplétude contractuelle liée à l'incertitude inhérente à la qualité des produits, l'auteur s'interroge sur les formes de coordination qui permettent néanmoins la production de l'accord entre le donneur d'ordres et le sous-traitant. L'auteur étudie tout d'abord les limites d'une gestion de la qualité des produits par le contrôle effectué par l'acheteur. Puis on montre que l'acquisition de la certification, analysée comme un signal, fonde la réputation de l'entreprise, cette réputation améliorant le fonctionnement du marché par la
\end{abstract}

\section{L'AUTEUR}

BERnARD BAUDRY est professeur agrégé à la Faculté de sciences économiques et de gestion de l'Université Lumière Lyon II et membre du Centre de recherches Auguste et Léon-Walras (ISH). Ses recherches portent actuellement sur la relation d'emploi et la théorie de la firme. Dernières publications : Économie Appliquée (1998) et Revue Économique (1999). Adresse: Faculté de sciences économiques et de gestion, 16, quai Claude-Bernard, 69007 Lyon (France) Téléphone: 3304786971 26. Courriel: bernard.baudry@univ-lyon2.fr

Je remercie les trois lecteurs anonymes de la revue pour leurs remarques et suggestions qu'ils ont adressées à une première version de ce texte. 
confiance que placent les acheteurs auprès des vendeurs. Par ailleurs, la qualité ne peut plus être reliée au seul produit, mais elle englobe la totalité de la relation interfirmes, et, de ce fait, la coordination se complexifie. En ce sens, la procédure de certification souffre de nombreuses limites et ne résume pas la totalité des mécanismes de coordination dans la relation de sous-traitance.

\begin{abstract}
In this paper, we try to analyse the industrial certification procedure in the subcontracting relationship. As the products market is caracterized by "incomplete contracts ", because of uncertainty about products quality, the author study the forms of coordination which permit agreement between buyers and suppliers. We first show the limits of the coordination based on products monitoring. Then we show that certification creates reputation for the subcontractor, and this reputation improves the functioning of the market. Finally, we show that the procedure of certification leads to a new approach of the quality, the products control being replaced by search of global quality in every element of the transaction between suppliers and buyers. In this sense, certification, because of its limits, is not the only mechanism of coordination in the subcontracting relationship.
\end{abstract}

\title{
RESUMEN
}

El fin de este artículo consiste en analizar el proceso de certificación industrial en la relación de segunda contratación. Saliendo de un contrato incompleto atado a la calidad de los productos, el autor se plantea problemas a propósito de los mecanismos de coordinación que permiten sin embargo la producción del acuerdo entre el comprador y el segundo contratante. El autor estudia primero los límites de una gestión de la calidad por el control que efectúa el comprador. Después, se muestra que la adquisición de la certificación vista como una señal produce la fama de la empresa, tal fama mejora el funcionamiento del mercado por la confianza que tienen los compradores en los vendedores. Además, la calidad no puede atarse exclusivamente al producto sino que comprende la totalidad de la relación entre las empresas. En ese sentido, el proceso de certificación da con muchos límites, y no resume la totalidad de los mecanismos de coordinación de la relación de segunda contratación.

\section{ZUSAMMENFASSUNG}

Dieser Artikel hat zum Ziel, den industriellen Zertifizierungsprozess im Zwischenhandel zu analysieren. Ausgehend von einer unvollständigen Vertragsausgangslage geknüpft an die unsichere Qualität der Produkte, hinterfragt der Autor den Prozess der Zusammenarbeitsformen betreffend den gegenseitigen Abkommen zwischen dem Auftragsgeber und dem Zwischenhändler. Der Autor untersucht die Limiten einer Qualitätskontrolle durch den Käufer. Anschliessend wird aufgezeigt, dass die Zertifizierung starken Einfluss hat auf das Unternehmensimage. Der gute Ruf stärkt das Käufervertrauen bei den Händler und trägt zur besseren Funktionalität des Marktes bei. Die Qualität ist nicht nur vom Produkt abhängig, sondern von den gesamten Unternehmensbeziehungen. Demzufolge wird die Koordination sehr komplex. In diesem Sinne ist der Zertifizierungsprozess im Zwischenhandel limitiert, denn die Zertifizierung umfasst somit nicht den ganzheitlichen Koordinationsmechanismus. 


\section{Introduction}

Depuis quelques années, une nouvelle approche de la qualité des produits est perceptible dans les relations interentreprises industrielles. En effet, jusqu'aux années 1980, la question de la qualité des produits n'était pas considérée comme majeure par les entreprises, dans la mesure où la croissance forte permettait d'écouler la production de biens relativement standardisés. Or, le ralentissement de cette croissance, les nouvelles formes de compétitivité, et notamment la concurrence des entreprises japonaises, ont entraîné une remise en cause de l'approche traditionnelle de la qualité dans les relations clients-fournisseurs, approche fondée essentiellement sur le contrôle. Celle-ci est désormais appréhendée par le biais de l'instauration de nouvelles procédures, de « manuels d'assurance qualité » susceptibles de certification, et elle s'inscrit dans un mouvement plus profond de réorganisation de l'ensemble des relations interentreprises, et notamment des relations grandes entreprises-PME.

Au point de vue théorique, on sait depuis l'article fondateur d'Akerlof (1970) que l'introduction de la qualité des biens perturbe fortement le modèle d'équilibre économique standard. Les analyses actuelles en termes d'asymétrie informationnelle montrent ainsi que l'inobservation du «niveau de qualité » du produit échangé par un acheteur est susceptible d'entraîner la carence du marché, voire sa disparition. L'économiste est alors conduit à s'interroger sur les différentes formes de coordination ${ }^{1}$ qui permettent malgré tout la production de l'accord sur la qualité des biens entre le client et le fournisseur.

L'objectif de cet article est donc d'analyser, d'un point de vue économique, la procédure de certification industrielle des PME et, plus précisément, des entreprises sous-traitantes, phénomène récent mais qui prend de plus en plus d'ampleur puisque tous les secteurs industriels sont aujourd'hui concernés par cette nouvelle approche de la qualité ${ }^{2}$. Notre problématique consistera à mettre en évidence le passage d'une définition de la qualité centrée sur le produit à celle d'une qualité dépassant les contours du seul produit. À cette modification de l'objet de la coordination, nous tenterons de montrer que les formes de coordination évoluent: d'une coordination fondée sur le contrôle du produit ex post, il y a passage à une coordination fondée, d'une part, sur la réputation ex ante du fournisseur, cette réputation étant gagée par les systèmes de certification, et, d'autre part, sur les liens interpersonnels.

1. Précisons que la coordination, au sens des dispositifs explicites ou implicites qui structurent la relation entre un acheteur et un vendeur, ne préjuge en rien du type de coopération-collaboration susceptible de s'instaurer entre ces mêmes acteurs.

2. Nous avons délibérément choisi de focaliser notre analyse sur la certification des soustraitants, car, comme nous le verrons dans la première section, c'est à propos de cette relation que les problèmes de «qualité » sont les plus aigus. Néanmoins, nous sommes conscient du fait que, dès lors, notre approche reste partielle. 
Dans une première section, nous préciserons le concept d'incomplétude contractuelle pour montrer qu'une incertitude sur la qualité d'un produit met en défaut une logique purement marchande. Deux types de marchés purs peuvent alors être opposés ; des «marchés de produit » et des « marchés d'organisation ».

Dans la deuxième section, nous verrons que la principale forme de coordination pour gérer l'incertitude sur la qualité des produits jusqu'aux années 1980 a reposé sur l'ensemble des contrôles mis en œuvre par le donneur d'ordres à l'égard des sous-traitants.

Compte tenu des limites d'une coordination fondée sur le contrôle, nous envisagerons alors la procédure de certification des PME sous-traitantes (section 3). En fait, cette procédure s'appuie sur la confiance des acheteurs à l'égard des vendeurs, confiance assise sur la réputation du sous-traitant, par l'intermédiaire de la certification qui permet aux acheteurs de porter un «jugement » sur le potentiel technologique et humain des vendeurs.

Dans une dernière section, nous tenterons de montrer qu'en fait les procédures de certification s'inscrivent dans un mouvement plus profond de réorganisation des relations interfirmes. Dans cette logique, la qualité ne peut plus être reliée au seul produit, mais elle englobe la totalité de la relation interfirmes, et, de ce fait, la coordination se complexifie. Nous verrons alors que la procédure de certification souffre de nombreuses limites et qu'elle ne résume pas la totalité des mécanismes de coordination dans la relation de sous-traitance.

\section{Incertitude et incomplétude contractuelle: marchés de produits et marchés d'organisation}

Pour apprécier le degré d'incertitude liée à la qualité des produits industriels, deux cas de figure possibles sont envisageables, qui sont fonction de la position du produit sur un axe allant du totalement standard au totalement sur mesure.

- Pour les produits standardisés, normés, il existe de nombreux fournisseurs potentiels, et le prix est alors le critère de décision le plus important. Pour ce type de produit, le risque encouru est faible, voire nul ; l'identité des parties est négligeable et la substituabilité des fournisseurs est forte. Un contrat classique d'achat-vente pour assurer une telle transaction est efficace. On dira alors que l'on se trouve en situation de complétude du contrat interfirmes (exemple du cas des matières premières) et on peut parler de «marché de produits » pour caractériser une telle situation (Favereau, 1989).

- La situation est différente pour les produits sur mesure (cas de la soustraitance et des sous-traitants fournisseurs comme les équipementiers 
automobiles) ${ }^{3}$. Ici, le produit n'est pas seulement une entité physique dotée d'utilités fonctionnelles, mais il inclut également la capacité du fournisseur à livrer en temps et lieu un produit parfaitement adapté au client, sa capacité à suggérer des innovations, à assurer un service de maintenance et d'assistance ${ }^{4}$. Autrement dit, se pose ici à l'acheteur le problème de l'antisélection puisqu'il est sous-informé sur une variable exogène, soit la qualité du produit échangé. Une pure logique marchande est donc prise en défaut dans la mesure où, par définition, le prix ne peut représenter un indicateur de la qualité, le produit n'étant pas disponible ex ante sur le marché. Si on adopte une perspective néo-institutionnaliste, on se rend compte que la recherche de l'information sur la qualité d'un produit, lorsque ce dernier n'est pas standardisé, engendre un coût de transaction qui perturbe fortement l'échange.

En d'autres termes, il n'y a plus ici identification du bien préalablement à l'échange. Le client, au moment du pacte initial avec son fournisseur, en $\mathrm{t}_{0}$, ne fait qu'acheter une potentialité et non un produit et seul le temps permettra de valider ou, au contraire, d'invalider le contrat initial. On est donc ici en situation d'incomplétude du contrat. Comme l'indique Eymard-Duvernay (1994), l'évaluation du client porte non sur un produit, mais sur « un potentiel, une capacité attachés à l'entreprise dont émane le produit ». On parlera alors de « marché d'organisation », toujours selon la terminologie de Favereau, les échanges étant caractérisés par l'incomplétude des contrats.

Dans le cadre de ce marché d'organisation, le client fait face à une double incertitude qui se déroule dans une perspective temporelle:

- il doit tout d'abord sélectionner en $\mathrm{t}_{0}$ un sous-traitant qu'il juge apte à réaliser une pièce sur mesure, qui, par définition, n'est pas disponible sur le marché. En effet, dans les relations de «quasi-intégration » (Houssiaux, 1957), l'originalité du produit sous-traité tient au fait qu'il est destiné à un seul demandeur. Salais note, à juste titre, que sur le marché anonyme, «l'identité de l'offreur et du demandeur n'importe pas dans le déroulement de la coordination » $(1994$, p. 384). En revanche, lorsque le produit est dédié et que les conditions de fabrication sont spécialisées, « on s'éloigne

3. Rappelons que la catégorie sous-traitants fournisseurs rassemble des entreprises qui conçoivent elles-mêmes leurs produits, compte tenu des spécifications fonctionnelles exigées par le client donneur d'ordres, alors que les sous-traitants se voient confier un cahier des charges strict et n'ont donc en principe aucun rôle actif dans la conception du produit (voir sur ce point Baudry, 1995).

4. En fait, c'est une définition très large de la qualité, mais nous verrons plus loin qu'elle correspond aux nouvelles formes de relations interentreprises, et notamment au «partenariat industriel». 
d'un produit objectif pour s'approcher du service rendu à une personne $A$ par une personne $B$, service qui tient sa valeur de tout ce qui fait que $A$ est $\mathrm{A}$ et que $\mathrm{B}$ est $\mathrm{B}$ » (Idem, p. 385).

- ensuite, entre $t_{0}$ et $t_{1}$ (remise du produit), il doit s'assurer que le vendeur entreprend les actions conformes à son intérêt. Or, la reconnaissance de l'opportunisme marchand fait que le vendeur est toujours susceptible de profiter d'un manque d'information de l'acheteur et d'entreprendre des actions inobservables qui augmentent la probabilité d'un défaut de qualité (Eymard-Duvernay, 1989).

La question de la qualité des biens constitue dès lors un point d'application central des réflexions en cours sur les logiques de coordination : comment assurer l'échange en situation d'asymétrie informationnelle? L'objectif des sections suivantes consiste justement, en nous appuyant sur les pratiques industrielles entre grandes entreprises et $\mathrm{PME}$, à réfléchir sur les différentes formes de coordination qui permettent de réduire l'incertitude liée à l'incomplétude du contrat.

\section{La coordination de la qualité des produits par le contrôle et ses limites}

Jusqu'aux années 1980, le contrôle par les donneurs d'ordres a constitué le dispositif principal de supervision de la qualité des produits et, d'un point de vue théorique, l'économie des coûts de transaction propose une grille de lecture pertinente de cette modalité de coordination (2.1.). Néanmoins, les limites de cette modalité sont progressivement apparues (2.2.).

\subsection{L'économie des coûts de transaction : l'intégration ou le contrôle comme dispositif de coordination ${ }^{5}$ ?}

La question du contrôle de la conformité du produit est cruciale dans la perspective de l'économie des coûts de transaction, puisqu'une des problématiques de ce courant consiste à analyser les décisions des firmes d'intégrer ou non certaines activités. S'interrogeant sur les motifs de l'intégration verticale, $M$. Ricketts suggère que « si la qualité des inputs est observable à faible coût, nous pouvons penser que des transactions de marché seront utilisées pour des achats récurrents d'articles standardisés » (Ricketts, 1987, p. 214). Or, le produit sous-traité n'étant pas par définition standardisé, le contrôle de la qualité est essentiel, mais il est extrêmement délicat à maîtriser. M. Casson établit une distinction pertinente entre les inspection goods et les experience goods (Casson, 1982, p. 171). Un produit à examiner est

5. Le contrôle correspond au terme «monitoring » utilisé dans la littérature anglo-saxonne. 
un produit dont la qualité peut être évaluée avant usage, tandis que le produit à expérimenter ne peut être évalué qu'à l'usage. Pour les premiers, le contrôle avant emploi n'évite pas, en cas de défectuosité, les coûts liés au fait d'avoir à trouver un article de remplacement. En revanche, l'utilisation d'un produit à expérimenter défectueux est susceptible de faire de graves dégâts monétaires. De plus, contrairement à l'achat d'un bien parfaitement standardisé, le produit sous-traité est unique et donc difficilement remplaçable.

Ricketts indique que cette difficulté d'évaluation peut être un motif d'intégration vers l'amont ou d'intégration modulée, l'une permettant de supprimer la situation d'information asymétrique avec le vendeur (1987, p. 215), l' autre produisant de l'information grâce à l'acquisition des compétences technologiques nécessaires à la vérification de la qualité.

L'accent mis sur le contrôle des produits pour assurer la coordination entre grandes entreprises et PME sous-traitantes constitue un éclairage pertinent pour analyser les relations interfirmes, mais indépendamment de la logique de l'intégration verticale, le client peut s'assurer de la conformité des produits en opérant des contrôles sur l'action du fournisseur. Cela suppose alors de reconnaître la capacité de la grande entreprise à s'ingérer dans la gestion de la PME.

Or, d'un point de vue empirique, et ce, jusqu'aux années 1980, le modèle dominant de la gestion de la qualité des produits, notamment dans les relations de sous-traitance (produits sur mesure) a en effet largement reposé sur le contrôle, seul dispositif de coordination. Ce dispositif résulte de la présence d'une «relation d'autorité » qui existe entre la PME et la grande entreprise (Baudry, 1995), et il s'exerce à la fois d'un point de vue purement contractuel (clauses de contrôle) et en marge du contrat. En effet, la subordination professionnelle du sous-traitant et sa dépendance économique permettent au donneur d'ordres d'effectuer des contrôles non prévus au contrat explicite. C'est donc en partie un «droit » pour ce dernier d'opérer chez son cocontractant ou sur l'objet du travail une série de vérifications.

Ce dispositif de coordination s'analyse donc bien comme une réponse au problème de l'information asymétrique, sans recourir à la stratégie de l'intégration verticale. Le contrôle constitue en fait un moyen qui permet de faire face à un comportement opportuniste éventuel du vendeur (par exemple, une tricherie sur la qualité).

D'une manière générale, le contrôle englobe toute l'exécution du contrat. Quatre principaux contrôles sont effectués par le donneur d'ordres :

- il existe tout d'abord les coûts liés à la prospection et à la sélection du soustraitant. Pour réduire la situation d'asymétrie informationnelle dont il souffre ex ante, le donneur d'ordres doit procéder à une évaluation de la 
capacité productive du sous-traitant. Les ateliers des sous-traitants sont ainsi visités, on leur confie des commandes tests, l'acheteur se renseigne sur la santé économique de l'entreprise du vendeur, sur sa «fiche sociale » (Gorgeu et Mathieu, 1983). Il s'agit ici pour l'acheteur de faire face à l'incertitude qui prévaut en $\mathrm{t}_{0}$;

- la pièce type est la deuxième manifestation du contrôle, et cette pièce est soumise à la vérification du client ;

- le client exerce ensuite des contrôles en cours de contrat, qui portent sur le travail lui-même et sur les conditions de la production (Gorgeu et Mathieu, 1983) ${ }^{6}$;

- le dernier contrôle concerne la conformité du produit : le donneur d'ordres agrée (ou non) le travail du sous-traitant.

On notera que ces trois derniers types de contrôle ont pour fonction de résoudre l'incertitude prévalant entre $t_{0}$ et $t_{1}$, c'est-à-dire celles qui concernent les conditions de la fabrication. Le modèle de gestion de la qualité est alors facilement caractérisable : il s'agit pour le client de s'assurer que le sous-traitant atteigne la norme fixée, cette norme correspondant à un palier minimum de caractéristiques techniques à respecter pour des raisons de commodité ou de sécurité.

\subsection{Les limites d'une approche de la qualité par le contrôle}

Ce mode de coordination fondé sur le contrôle a progressivement présenté des limites, notamment à partir de la fin des années 1970, pour les raisons suivantes :

- le contrôle est un dispositif coûteux pour le client ;

- il ne peut empêcher le fournisseur de «biaiser» sur la qualité, soit intentionnellement, soit inintentionnellement si l'appareil de production n'est pas suffisamment performant ;

- le contrôle porte essentiellement sur le produit et non sur le potentiel technologique du fournisseur. Or, les défauts des produits sont fortement liés aux défauts des processus situés en amont, dès la conception;

- enfin, ce mode de coordination est totalement incompatible avec les nouvelles techniques de livraison qui s'instaurent depuis les années 1980

6. Gorgeu et Mathieu soulignent, dans le compte rendu de leur enquête de 1983, que «la vérification en cours d'exécution est très fréquente parmi les entreprises donneuses d'ordres étudiées, puisque presque toutes envoient des contrôleurs chez leurs sous-traitants soit de façon régulière, soit de façon imprévue [...] Le contrôle en cours d'exécution du travail apparaît comme un élément de plus du "droit de regard" que le donneur d'ordres veut exercer sur ses sous-traitants ». 
entre les donneurs d'ordres et les sous-traitants. On constate en effet le passage d'une gestion de l'organisation physique de la production par les stocks à une gestion à «flux tendus ». Ce système consiste à livrer « justeà-temps », c'est-à-dire au moment précis où les besoins se manifestent. L'objectif est évidemment de diminuer l'importance des stocks et donc de recevoir les pièces et les composants le moins longtemps possible avant leur utilisation. C'est dans la construction automobile que la pratique des livraisons en juste-à-temps entre grandes entreprises et PME est la plus développée (Gorgeu et Mathieu, 1994). La forme la plus tendue est la livraison en flux synchrones : l'ordre de livraison est envoyé par télétransmission par l'usine de montage du constructeur au moment où chaque caisse de voiture identifiée par caméra entre dans l'atelier de montage. Le fournisseur dispose alors de quelques heures pour livrer dans l'ordre les produits correspondant à chaque voiture. Dans ces conditions, le produit n'est plus contrôlé à l'arrivée et il doit donc répondre totalement aux spécifications du client, ce qui implique la nécessité d'un autre dispositif de coordination entre la PME et ses clients pour « assurer » la qualité des produits.

\section{La procédure de certification : une approche en termes de réputation et de confiance}

Du fait de son coût et de ses insuffisances, la coordination par le contrôle est progressivement remplacée par une coordination fondée sur «l'assurance de la qualité ».

Concrètement, le « système d'assurance de la qualité » consiste à formaliser par écrit tout ce qui se fait dans l'entreprise, c'est-à-dire que l'entreprise doit décrire dans un recueil de documents le système qui lui permet d'obtenir et de garantir le niveau de qualité requis (Cung, 1993). Lorsqu'une entreprise dispose d'un système d'assurance qualité, elle peut ensuite demander la certification de ce système.

La certification d'entreprise est une procédure qui atteste la conformité du système d'assurance qualité d'une entreprise à l'un des trois modèles normalisés de la série des normes ISO 9000 (la norme 9001 porte sur l'assurance qualité de la conception au service après-vente ; la norme 9002 ne traite pas de la partie conception ; la norme 9003 concerne seulement le contrôle des produits en sortie d'usine) ${ }^{7}$. L'AFAQ (Association française d'assurance pour la qualité) est l'organisme

7. La norme 9004 , quant à elle, constitue un guide d'utilisation pour la gestion de la qualité et la mise en place d'éléments du système qualité et ne peut pas faire l'objet d'une certification. Par ailleurs, d'ici l'an 2000, les normes ISO 9001, 9002 et 9003 doivent être refondues en une seule norme plus ouverte vers la qualité totale. 
principal qui délivre en France le certificat à la suite d'un audit permettant de vérifier l'existence chez le fournisseur ou le sous-traitant d'une organisation conforme à l'ensemble des normes définissant l'assurance de la qualité ; de plus, pendant la durée de validité du certificat (trois ans), l'entreprise est soumise à plusieurs visites de suivi. En 1995, environ 5000 certificats ont été délivrés, et un certificat sur cinq porte sur la norme $9001^{8}$. Comment analyser d'un point de vue théorique ce mouvement croissant de certification?

Nous proposons en fait de replacer la procédure de certification dans un contexte plus large de construction de la confiance entre le donneur d'ordres et son sous-traitant, par l'intermédiaire du concept de réputation. Introduire le concept de confiance semble effectivement logique dès lors qu'il existe entre les acteurs une incertitude pesant sur l'échange, de même que sur la qualité d'un produit. Nous verrons successivement le fonctionnement du marché avec réputation sans certification, puis avec certification.

En l'absence de certification, les croyances des acheteurs sur la fiabilité des vendeurs à fournir la qualité exigée (faire ou ne pas faire confiance) sont basées sur le comportement passé des firmes en matière de qualité. La qualité obtenue lors des transactions précédentes indique le niveau de qualité que l'on peut obtenir dans le futur.

Ainsi, dans un jeu indéfiniment répété entre X (l'acheteur) et Y (le vendeur), si $\mathrm{Y}$ fait défection une fois (il trahit la confiance) alors $\mathrm{X}$ se venge en décidant de ne plus coopérer avec $\mathrm{Y}$, qui, de ce fait, perd définitivement les gains issus d'une éventuelle relation à long terme avec $\mathrm{X}$, et, de surcroît, la réputation de $\mathrm{Y}$ s'effondre et $\mathrm{Y}$ se prive de relations d'affaires avec des partenaires potentiels. La réputation s'acquiert ainsi en ne déviant jamais de la coopération ${ }^{9}$. C'est donc en misant sur l'intérêt de l'autre que l'on force le contrat, grâce à la menace d'infliger des pertes supérieures aux gains de court terme issus de la tricherie - au contractant. Le contrat devient alors auto-exécutoire (self-enforcing) puisque chaque partenaire a intérêt à respecter les termes de ce contrat implicite. Cela revient à dire que le fournisseur livrera systématiquement un bien avec la qualité promise, pour ne pas détruire son capital de réputation. On peut ainsi penser qu'un sous-traitant qui dégrade la qualité de ses produits à une période donnée sera exclu du marché de la sous-traitance à la période suivante, ce qui doit donc théoriquement l'inciter à ne pas dévier du choix coopératif.

Cette problématique n'est cependant pas sans comporter des limites :

- notons tout d'abord le caractère purement bilatéral de cette interaction. Dès lors, la réputation de $\mathrm{Y}$ doit être visible, ou, à défaut, transmissible, pour

8. Chiffres cités dans Industries, no 3, décembre-janvier 1995.

9. «Coopération» au sens de la théorie des jeux. 
que des agents $X_{i}$, qui n'ont jamais interagi auparavant avec $Y$, décident de rentrer en relation avec $\mathrm{Y}$, en s'appuyant sur la réputation de $\mathrm{Y}$. Or, cette transmission ne va pas de soi, Eymard-Duvernay soulignant à juste titre que la réputation « suppose des outils de diffusion des informations. Compte tenu de ce coût, le passage à des transactions entre des individus en grand nombre n'est pas immédiat. Bien souvent, la réputation ne sera effective que sur des réseaux individuels limités » (1994, p. 316). Autrement dit, une des limites de ce modèle de réputation sans certification réside dans le fait qu'on ne tient pas compte des objets dans la coordination. Or, nous verrons plus loin l'importance des processus de certification, gagée sur le niveau des équipements du fournisseur, pour assurer la coordination et garantir le contrat ;

- dans cette configuration, où seul le produit est évalué (l'output), on ne fait pas cas du potentiel de l'entreprise (le comportement des salariés de Y et le niveau des équipements). Or, comme nous l'avons noté plus haut, les défauts des produits sont fortement liés aux défauts des processus situés en amont, dès la conception;

- enfin, ce modèle suppose que dans le cours de l'interaction, les partenaires soient en mesure de vérifier si les engagements initiaux ont été respectés, autrement dit que Y n'a pas triché - sur la qualité. Or, Y peut avoir triché intentionnellement ou alors la défection peut s'avérer inintentionnelle. Autrement dit, le risque de défaut est bien évidemment lié à l'honnêteté du contractant, mais il dépend également du degré de compétence du fournisseur Y, ou encore de variations naturelles dans le processus de production. La réputation représente, dans ce cas, un dispositif insuffisant dans la mesure où la défaillance du fournisseur Y n'est pas nécessairement liée à une volonté délibérée d'adopter un comportement opportuniste.

On peut maintenant s'interroger sur l'avantage lié à l'introduction de la certification par rapport au fonctionnement d'un marché sans certification.

Ce qui fonde dans ce cas de figure la confiance de l'acheteur, c'est sa capacité à obtenir des informations sur le cocontractant (information ex ante) et non sur la qualité d'un produit (information ex post), informations qui ont pour propriété de diminuer l'incertitude inhérente au contrat conclu ex ante. D'ailleurs, c'est bien ainsi que l'envisagent les praticiens, puisque l'assurance qualité recouvre «l'ensemble des actions préétablies et systématiquement nécessaires pour donner la confiance appropriée en ce qu'un produit ou service satisfera aux exigences relatives à la qualité » (AFNOR $\left.{ }^{10}, 1992\right)$. Nous proposons ainsi d'envisager la réputation lorsque les vendeurs sont en mesure de signaler leur véritable qualité.

32. Association française de normalisation. 
En ce qui concerne la théorie du signal, rappelons que lorsqu'il existe une situation d'asymétrie informationnelle sur un marché, les bons agents - ceux qui vendent un produit de «bonne qualité »-sont pénalisés par rapport aux mauvais agents puisqu'ex ante il est impossible pour l'acheteur de séparer les deux types d'agents. Les bons agents ont alors tout intérêt à signaler leur qualité (Spence, 1974) ${ }^{11}$. On sait par ailleurs que le signal est d'autant plus crédible qu'il est coûteux (et plus coûteux pour les vendeurs de produits de mauvaise qualité [McMillan, 1992]) ou qu'il est garanti par un tiers. Autrement dit, il ne faut pas que les agents qui ont des biens de mauvaise qualité aient intérêt à envoyer un faux signal (Cahuc, 1993).

La certification constitue un point d'application évident de ce cadre théorique, puisqu'elle représente un actif qui sert de signal à l'acheteur pour effectuer son jugement sur la fiabilité du vendeur (sa capacité à honorer le contrat), et ce dernier fera confiance à une entreprise certifiée, car elle est supposée fiable ex ante. De plus, ce signal est incontestablement plus coûteux pour les PME dont le niveau technique des équipements est faible, puisque obtenir la certification pour ces entreprises suppose une démarche longue et des efforts d'investissement et de modernisation importants.

En outre, ce signal est également crédible s'il est garanti par un tiers. Or, dans le cas des certifications interentreprises, il existe deux catégories de certification, une certification effectuée par le client ${ }^{12}$ et une certification effectuée par un organisme indépendant, comme l'AFAQ. Cette distinction peut avoir des implications importantes suivant le type d'entreprise qui effectue la certification. A priori, une certification effectuée par un client $\mathrm{X}$ a par définition un domaine de validité limitée : un fournisseur Y certifié par cette entreprise $X$ ne pourra se prévaloir de cette certification auprès d'un client $\mathrm{Z}$ qui n'a peut-être pas les mêmes critères de jugement de la qualité. La confiance restera alors purement bilatérale dans ce cadre. Néanmoins, la certification bilatérale constitue un véritable signal de qualité susceptible de se diffuser auprès des autres contractants lorsque l'entreprise certificatrice possède elle-même une réputation de crédibilité. C'est le cas par exemple des certifications accordées par les constructeurs automobiles, dans la

11. On trouvera un modèle formalisé de réputation avec signal dans le cas d'un marché avec asymétrie d'information sur la qualité dans Coestier, 1995. L'auteur analyse notamment les conséquences des tests de qualité sur la fixation du prix des produits. Voir également Kreps (1990) pour l'étude de la réputation.

12. Les gestionnaires parlent dans ce cas de «qualification », dans la mesure où l'agrément du système qualité est accordé par une entreprise et non par un organisme indépendant comme l'AFAQ (voir Cung, 1993). 
mesure où l'ensemble de la communauté des donneurs d'ordres sait que leurs critères sont parfois encore plus exigeants que ceux des normes ISO (Gorgeu et Mathieu, 1996) ${ }^{13}$.

Dans le cas de la certification AFAQ, le client s'en remet à un tiers extérieur pour fonder son jugement ${ }^{14}$, tiers lui-même crédible car reconnu et accepté par l'ensemble des donneurs d'ordres, et cette certification permet d'élargir les relations d'affaires potentielles du sous-traitant certifié.

Dès lors, la procédure de certification fonde la réputation du vendeur, mais ici, la réputation n'est pas engendrée par la répétition de l'échange, contrairement au modèle précédent (sans certification) : elle s'appuie sur des objets, sur l'équipement de l'entreprise, sur sa capacité à assurer un niveau de qualité reproductible.

L'origine latine du terme réputation est d'ailleurs à cet égard particulièrement éclairante : réputio signifie «supputer, calculer, compter». Or, dans le cas de la procédure de certification, il s'agit bien d'évaluer les compétences des vendeurs, leur capacité à être fiables. On comprend alors l'intérêt de la certification par un tiers extérieur: la réputation est incontestable car gagée par un tiers extérieur, et cette réputation, contrairement au modèle précédent, peut être diffusée, transférée à l'ensemble des clients de la profession. Autrement dit, si une entreprise $Y$ est certifiée par l'AFAQ, des agents $X_{i}$ qui n'ont jamais interagi avec $Y$ peuvent lui faire confiance. La réputation n'est donc pas ici produite par la durée ou par la répétition de l'échange ; elle est extérieure aux individus qui opèrent dans l'organisation, elle est détachée des personnes et un individu qui quitte cette organisation ne remet pas en cause la réputation de l'entreprise, contrairement à des transactions qui reposent sur la «qualité » et la singularité des personnes. Notons que le raisonnement est identique pour une certification bilatérale, à condition, comme nous l'avons indiqué plus haut, que l'entreprise certificatrice soit crédible ${ }^{15}$.

Quelles sont alors les propriétés de cette réputation fondée sur la certification?

Une firme qui ne respecterait pas les procédures de l'assurance qualité et qui détériorerait délibérément la qualité des produits perdrait instantanément sa réputation (il existe des visites de suivi et le certificat est valable seulement trois ans) et cette perte de réputation serait immédiatement diffusée parmi les acheteurs potentiels, contrairement à la logique du modèle de réputation sans certification.

13. Gorgeu et Mathieu notent que l'évaluation d'aptitude qualité fournisseurs (EAQF) des constructeurs de 1990 allait déjà au-delà des normes ISO 9002 et même 9001 (1996, p. 228).

14. On fera ici l'hypothèse que le client a confiance dans l'organisme qui délivre le certificat.

15. Les sous-traitants fournisseurs classés en catégorie A par les constructeurs automobiles peuvent ainsi se prévaloir de ce classement auprès d'autres donneurs d'ordres. 
La certification crée ainsi des obligations pour le sous-traitant, ce qui doit l'inciter à respecter dans le temps les clauses du contrat, sous peine de voir son capital fondre, se déprécier. Conserver le certificat, c'est alors pour l'entreprise adopter un comportement loyal, donc ne pas faire défection (ne pas trahir la confiance) et le contrat peut dès lors devenir auto-exécutoire. Ce comportement loyal est en définitive garanti par l'observation scrupuleuse des principes, modes opératoires et procédures qui sont formalisés dans le manuel qualité. Ce raisonnement suppose évidemment que les gains potentiels tirés de la procédure de certification (répétition de l'échange avec les acheteurs) soient supérieurs à son coût, coût lié, d'une part, au niveau des équipements de l'entreprise en $t_{0}$ et, d'autre part, aux investissements qui sont nécessaires pour conserver la certification en $\mathrm{t}_{1}, \mathrm{t}_{2}$, etc.

Ensuite, par rapport au modèle de réputation sans certification, notons que la procédure de certification permet d'améliorer la fluidité du marché et de diminuer les coûts de transaction, dans la mesure où l'information sur le cocontractant est immédiatement disponible, aucune période d'apprentissage n'étant requise pour valider la croyance de l'acheteur en ce qui concerne la réputation du vendeur. Autrement dit, un client peut immédiatement entrer en relation avec un sous-traitant certifié avec qui il n'a jamais contracté.

Plus largement, et nous y reviendrons en conclusion, cette procédure représente une incitation au dynamisme et à la modernisation pour les entreprises, la performance en termes de qualité étant une nécessité pour conserver la certification.

Néanmoins, il nous semble que cette approche théorique de la certification par la réputation n'épuise pas la totalité des modifications en cours. En effet, la certification représente seulement la partie visible de l'iceberg, car cette procédure s'intègre plus généralement dans une nouvelle approche de la qualité.

\section{Les limites de la certification comme dispositif de coordination : la nouvelle approche de la qualité}

Indépendamment des nouvelles formes de livraison (en flux tendus) dont nous avons parlé précédemment, deux autres modifications essentielles altèrent la relation de sous-traitance :

- la conception du produit est désormais largement partagée entre clients et certains sous-traitants, notamment dans l'automobile (Gorgeu et Mathieu, 1994). Dans cette nouvelle relation, l'innovation du vendeur est sollicitée et ses compétences s'élargissent, le vendeur étant désormais chargé de la réalisation de sous-ensembles, de fonctions complètes ;

- la relation se matérialise fréquemment par la passation de contrats, explicites ou implicites, qui introduisent la durée dans la relation. 
Ces trois éléments permettent de qualifier ce que l'on appelle la logique partenariale, logique qui modifie l'organisation interfirmes - la relation de soustraitance classique -, une quasi-intégration oblique se substituant dans certains cas à la quasi-intégration verticale (Baudry, 1995).

Dès lors, dans une telle relation, la définition de la qualité s'enrichit, car ce n'est plus seulement le produit qui est en cause dans la coordination, mais un ensemble beaucoup plus large qui densifie cette relation : conception du produit, conditions de livraison, innovations, analyse de la valeur, etc. ${ }^{16}$. On retrouve ici la logique qui caractérise les marchés d'organisation, cette nouvelle approche de la qualité se substituant, en tant qu'objet de coordination, à l'ancienne définition de la qualité centrée sur le seul produit.

En ce sens, cette nouvelle approche de la qualité modifie la double situation d'incertitude que nous avons présentée au début de ce travail : ce n'est plus la qualité du produit au sens strict qui constitue l'objet de la coordination, mais l'incertitude qui se situe d'une part en amont, en $\mathrm{t}_{0}$, sur la capacité du sous-traitant à innover, à proposer des solutions techniques, et d'autre part en aval, en $t_{1}$, c'est-à-dire sur sa capacité à livrer en juste-à-temps (et non plus sur sa capacité à livrer un produit conforme). En revanche, la certification allège les conditions de la transaction entre $\mathrm{t}_{0}$ et $\mathrm{t}_{1}$, c'est-à-dire lors des opérations de fabrication du produit, la réputation rendant le contrat auto-exécutoire. Autrement dit, notre découpage temporel de la relation de sous-traitance montre que la certification se révèle nettement insuffisante pour lever l'incertitude qui pèse sur la transaction et pour engendrer une véritable coopération entre les entreprises. On peut alors s'interroger sur les différentes formes de coordination qui correspondent à cette nouvelle approche de la «qualité ».

A priori, l'émergence et le développement progressif des normes ISO semblent avoir comme conséquence, d'une part, la réduction de la place du marché (rôle du prix) et, d'autre part, la diminution de la présence des liens interpersonnels dans la coordination.

En effet, d'un point de vue théorique, la certification signifie un glissement d'une coordination marchande vers une coordination plus industrielle : le prix n'est pas (plus) la variable unique de la transaction. Pour autant, peut-on conclure à son

16. Cette nouvelle acception du terme «qualité » est d'ailleurs largement reconnue par les praticiens puisque pour l'AFNOR « la qualité d'un produit s'analyse en divers éléments : les multiples caractéristiques, l'esthétique, la sûreté de fonctionnement, la sécurité, l'ergonomie, le respect des délais, la documentation d'emploi et de maintenance, le coût de possession, l'information sur les produits et sur l'entreprise, la facturation et les conditions de paiement, l'anticipation sur l'évolution des besoins des utilisateurs...» (1992, p. VII). Certains praticiens parlent alors de «qualité totale » (voir Le Monde du 14 décembre 1994, p. 6). 
élimination dans le processus concurrentiel ? En fait, certaines études empiriques (Gorgeu et Mathieu, 1994) permettent de nuancer ce jugement. En effet, la normalisation de la qualité, sanctionnée par les procédures de certification, a comme propriété de rendre directement comparables entre eux différents produits qui satisfont un même besoin. La certification, par la reconnaissance des référentiels de qualité qui sont imposés aux entreprises, aboutit ainsi à une mesure commune de la qualité. Pour reprendre la typologie établie par Salais en termes de produit spécialisé et dédié, la certification fait disparaître l'aspect spécialisé du produit pour le transformer en produit standard, c'est-à-dire un produit dont les normes de fabrication sont en partie codifiées et qui donc ne donne au producteur «aucune possibilité de signifier son identité sur le produit » (1994, p. 385). En ce sens, comme nous l'avons déjà noté, la certification permet au marché d'être plus transparent, ce qui peut redonner de la vigueur aux lois du marché et rendre au prix son rôle de régulateur. À cet égard, notons qu'une généralisation des procédures de certification à long terme entraînera vraisemblablement une dévaluation de ce dispositif de coordination en tant que facteur de différenciation des entreprises ${ }^{17}$, les dispositifs marchands retrouvant alors toute leur intensité lors de la sélection du fournisseur.

En outre, si la certification rend théoriquement inutile toute coordination par les liens interpersonnels, puisque la normalisation est censée régler la question de la qualité, dans la pratique, la nouvelle définition de la qualité, les nouvelles formes de coopération grandes entreprises-PME sous-traitantes, s'accompagnent d'une intensification des engagements personnels entre acheteurs et vendeurs, notamment pour lever l'incertitude qui prévaut en amont et en aval. La collaboration interentreprises est ainsi efficace en matière d'innovation de produits et d'analyse de la valeur, à condition que l'information soit partagée. La coopération interfirmes permet, lorsqu'une réelle réciprocité s'instaure dans l'échange d'informations, de prévoir l'évolution de la demande. Par exemple, dans le secteur automobile, de nombreux équipementiers, en liaison avec les constructeurs automobiles, réfléchissent à la manière d'anticiper les évolutions technologiques et proposent des solutions et des innovations. Un tel apprentissage fournit une information commune et un langage propre à la relation interorganisationnelle (Pernin, 1993). Parallèlement, les procédures de mise au point des techniques de livraison à flux tendus sont très complexes et nécessitent un transfert d'information dense et une codification rigide.

Finalement, on se rend compte que la gestion de cette nouvelle approche de la qualité repose sur une pluralité de dispositifs de coordination, pluralité qui est fonction du type de relation existant entre l'acheteur et le vendeur. Dans une relation de type quasi-intégration oblique, la coordination de la qualité s'appuiera largement sur la réputation (au regard de la certification) et sur les actifs spécifiques humains

17. On peut évidemment penser ici au processus qui concourt à la dévaluation des diplômes. 
engagés dans la transaction pour innover et améliorer les produits. En revanche, dans une relation de type quasi-intégration verticale, la coordination se rapprochera plus du pôle marchand, la qualité constituant une variable moins déterminante dans la relation. Autrement dit, la procédure de certification ne représente en aucun cas une solution «miracle » capable à elle seule de régler les questions liées à la définition de la qualité dans la relation de sous-traitance.

\section{Conclusion}

On le constate, l'approche de la qualité basée sur la certification, malgré les limites de cette procédure, remet profondément en cause la structuration de l'organisation industrielle et transforme les logiques de coordination interentreprises.

Indépendamment des modifications concernant les modalités de coordination interfirmes, nous mentionnerons dans cette conclusion trois conséquences de la procédure de certification en cours sur les relations grandes firmes - PME soustraitantes :

- tout d'abord, la certification transforme la structuration de l'organisation industrielle, dans la mesure où les entreprises non certifiées sont exclues comme fournisseurs directs des grandes entreprises. Par exemple, dans l'industrie automobile, la procédure de certification a entraîné (et ce mouvement continue) une diminution considérable du nombre de fournisseurs directs. Renault et PSA ont ainsi instauré, à partir des années 1980, une politique similaire de réduction du nombre de leurs fournisseurs directs. En 1980, la filiale de PSA centralisant les achats du groupe, la SOGEDAC, avait, pour les usines PSA en France, 2000 fournisseurs directs pour les pièces de série ; elle n'en avait plus que 985 huit ans plus tard et $770 \mathrm{au}$ $1^{\text {er }}$ janvier 1990, et 700 environ en 1994. Il en est de même pour Renault ; cette entreprise avait 1415 fournisseurs directs en 1985, 900 en $1989^{18}$.

L'évolution est identique dans le secteur aéronautique puisque l'Aérospatiale, avant la restructuration de ses activités, comptait 650 sous-traitants, et aujourd'hui environ 200, le processus de sélection n'étant pas encore terminé (Morin, 1994).

Pour ces deux secteurs, les critères de choix des fournisseurs «privilégiés » sont quasiment identiques : capacité à répondre aux normes de qualité imposées par le constructeur, aptitude à livrer les quantités demandées en flux tendus (pour le secteur automobile), aptitude à participer au développement technique, compétitivité sur les prix, situation financière saine (Gorgeu et Mathieu, 1994 ; Morin, 1994). Autrement dit, la certification opère bien comme mode de classement des entreprises.

18. Chiffres cités par la revue Enjeux, no 148 , octobre 1994. 
- ensuite, en ce qui concerne les relations de travail internes, certains gestionnaires pensent que les procédures d'assurance qualité et leur certification contribuent à la modernisation de l'entreprise et à l'élévation du niveau de qualité. Ce serait ainsi l'occasion pour l'entreprise de construire un véritable « projet d'entreprise » et d'élever les compétences, le niveau de formation et de qualification des salariés (Segrestin, 1996). C'est également pour les partisans des normes ISO la possibilité de responsabiliser les salariés, notamment par l'exigence de « traçabilité » qui permet de remonter à la source des dysfonctionnements. La formalisation et l'établissement de procédures écrites permettent également de transférer plus rapidement des personnels d'un poste à un autre, économisant sur les procédures d'apprentissage liées à une fonction déterminée. Plus généralement, il semble que la procédure de certification et la nouvelle approche de la qualité constituent une incitation au dynamisme et à la modernisation pour les PME. Gomel, Gorgeu et Mathieu (1992), en s'appuyant sur une étude empirique, notent ainsi que la satisfaction des exigences de la qualité et l'élargissement des compétences requièrent des investissements spécifiques et des investissements en formation du personnel. Autrement dit, il est incontestable que la certification, en tant que procédure de coordination, améliore la compétitivité des entreprises ;

- enfin, on peut se demander si la procédure de certification ne remet pas en cause la relation d'autorité qui existe entre les grands groupes et les PME sous-traitantes. En effet, cette nouvelle approche de la qualité modifie la situation de dépendance de la PME, dans la mesure où la firme certifiée voit ses compétences s'élargir en termes de qualification et de formation, et un des critères qui fondent la relation d'autorité, la dépendance économique, s'affaiblit, la PME sous-traitante étant alors à même de conquérir de nouveaux clients et de nouveaux marchés.

\section{Bibliographie}

AFNOR (1992), Gérer et assurer la qualité, tome 2, Paris, Association française de normalisation, $344 \mathrm{p}$.

AKERLOF, G. (1970), «The market for "Lemons" : qualitative uncertainty and the market mechanism », The Quarterly Journal of Economics, vol. 84, p. 488-500.

BAUDRY, B. (1995), L'économie des relations interentreprises, Paris, La Découverte, Collection « Repères ».

CAHUC, P. (1993), La nouvelle microéconomie, Paris, La Découverte, Collection « Repères ». CAsson, M. (1982), The Entrepreneur : An Economic Theory, Totowa, Barnes and Noble. COESTIER, B. (1995), «Asymétrie d'information, réputation et signes de qualité », CEPREMAP, Document de recherche ${ }^{\circ} 9505$. 
CunG, D. (1993), «La certification : mode ou nécessité ? », Gestion 2000, vol. 4, p. 13-24.

EYMARD-Duvernay, F. (1989), "Conventions de qualité et formes de coordination », Revue économique, vol. 40, $\mathrm{n}^{\circ}$ 2, mars, p. 329-359.

EYMARD-DUVERNAY, F. (1994), «Coordination des échanges par l'entreprise et qualité des biens », dans A. Orléan (dir.), Analyse économique des conventions, Paris, Presses universitaires de France, p. 307-334.

FAVEREAU, O. (1989), « Marchés internes, marchés externes », Revue économique, $\mathrm{n}^{\circ} 2$, mars, p. 273-328.

Gomel, B., A. GorgeU et R. MAthiEU (1992), «Les nouvelles exigences des donneurs d'ordres : une incitation au dynamisme pour les sous-traitants ?», CEE, Dossier de recherches $n^{\circ} 45$, mars.

Gorgeu, A. et R. MAthieu (1983), « Sous-traitance et emploi », Cahiers $d u C E E, \mathrm{n}^{\circ} 23$.

GORGEU, A. et R. MATHIEU (1994), «Le partenariat entre constructeurs automobiles et équipementiers : les frontières de la firme ?», CEE, Document de travail, mai.

GoRGEU, A. et R. MATHIEU (1996), «L'assurance qualité fournisseur de l'industrie automobile française », Revue d'économie industrielle, $\mathrm{n}^{\mathrm{0}} 75,1^{\mathrm{er}}$ trimestre, p. 223-238.

GRENARD, A. (1996), «Normalisation, certification : quelques éléments de définition », Revue d'économie industrielle, $\mathrm{n}^{\mathrm{o}} 75,1^{\mathrm{er}}$ trimestre, p. 45-60.

HoussiauX, J. (1957), «Le concept de quasi-intégration et le rôle des sous-traitants de l'industrie », Revue économique, mars, p. 221-247.

KREPS, D. (1990), «Corporate culture and economic theory », dans J. Alt et K. Shepsle (dir.), Perspectives on Political Economy, Cambridge, Cambridge University Press.

MCMillan, J. (1992), Games, Strategies and Managers, New York, Oxford University Press.

MORIN, M.L. (1994), « Sous-traitance et relations salariales : aspects de droit du travail », CEJEE, Université des Sciences sociales de Toulouse.

PERNIN, J.L. (1993), «La coopération entre firmes : une approche par l'économie des conventions », Économie appliquée, tome XLVI, no 4, p. 105-126.

RAVIX, J.T. et P. ROMANI (1996), "Certification et formes de coordination dans l'organisation de la production industrielle », Revue d'économie industrielle, $\mathrm{n}^{\circ} 75$, $1^{\text {er }}$ trimestre, p. 275-290.

RICKETTS, M. (1987), The Economics of Business Enterprise : New Approaches to the Firm, New York, Harvester Wheatsheaf.

SALAIS, R. (1994), «Incertitude et interactions de travail : des produits aux conventions », dans A. Orléan (dir.), Analyse économique des conventions, Paris, Presses universitaires de France, p. 371-403.

SEgRESTIN, D. (1996), «La normalisation de la qualité et l'évolution de la relation de production », Revue d'économie industrielle, $\mathrm{n}^{\mathrm{0}} 75,1^{\mathrm{er}}$ trimestre, p. 291-307.

Spence, M. (1974), Market Signaling, Cambridge, Harvard University Press.

STIGLITZ, J.E. (1987), «The causes and consequences of quality on price», Journal of Economic Literature, vol. 25, p. 1-48.

WILLIAMSON, O.E. (1985), The Economic Institutions of Capitalism, New York, Free Press. 